\title{
Las Habilidades Blandas Factor Clave Para El Desempeño De Los Ingenieros
}

\author{
Rubén Gómez Sánchez Soto \\ Universidad Nacional de Ingeniería, Lima, Perú
}

Recibido: 15/04/2021 Revisado: 21/05/2021 Aceptado: 26/06/2021 Publicado: 28/07/2021

\section{RESUMEN}

El trabajo analizó los impactos de la no consideración de las habilidades blandas en los programas de formación de los nuevos ingenieros.

El diseño de la investigación fue cualitativo y correlacional teniendo como objetivo determinar la correlación de la no consideración de las diferentes habilidades blandas en el desempeño de los ingenieros, en sus diferentes facetas de trabajo.

Según James Albert Michener: "Los científicos sueñan con hacer grandes cosas; los ingenieros simplemente las hacen" (Tribuno, 2020). La investigación se inició evaluando el rol de la ingeniería en la sociedad.

A continuación, se analizó la definición de las habilidades blandas y sus aplicaciones, la necesidad de éstas en la formación de los ingenieros, el rol de éstas en el ejercicio de la gestión de proyectos, y finalmente su función en el desempeño gerencial de los ingenieros. Estas son las diferentes facetas en las cuales los ingenieros desarrollan su ejercicio profesional.

Según los resultados alcanzados la ingeniería es responsable de los avances de la sociedad, pero para responder a estos enormes retos se observó el rol de las habilidades blandas en su proceso de formación; ya que, cubriendo satisfactoriamente este reto, se podría responder mejor a los retos de la sociedad.

Palabras claves: Retos de ingeniería, Habilidades duras, Habilidades blandas, Inteligencia emocional, éxitos en los proyectos. 


\section{INTRODUCCIÓN}

El problema que motivó el presente trabajo fue: "Analizar el papel de las habilidades blandas en el desempeño de los ingenieros" y como esta gama de habilidades podrían generar impactos en los resultados del ejercicio profesional de los ingenieros en ejercicio y de los nuevos ingenieros. Esta preocupación cobra más importancia por el rol de los ingenieros ante la sociedad.

Los objetivos de la investigación realizada podrían sustentar la revisión de los programas actuales de formación de los nuevos ingenieros, en cuanto a la necesidad de incorporación de las habilidades blandas, y como éstas podrían a mejorar el desempeño de los ingenieros. Y por supuesto establecer recomendaciones para pensar y reflexionar en mejora de tales programas de formación.

El Perú cuenta con una seria de antecedentes respecto de programas de formación de los ingenieros.

Los modelos educativos en uso y su relación directa con la problemática de desempeño de los profesionales de ingeniería, sustentaría la existencia de brechas, fortalezas, y problemática para responder a las expectativas de la sociedad, respecto del ejercicio profesional de la ingeniería.

\section{METODOLOGIA}

\subsection{Rol de la ingeniería en la sociedad}

La ingeniería es una profesión que tiene como misión el bienestar de la sociedad; por lo que, se considera necesario plantear una propuesta de misión de los ingenieros: "La ingeniería debe tomar como suyos los problemas de la sociedad, plantear soluciones, en las cuales emplea materiales y las fuerzas de la naturaleza, elige las más satisfactoria bajo todo punto de vista y principalmente el económico. Debe concretar la solución, resolver los problemas de la sociedad, y lograr la satisfacción de las necesidades humanas". Esta propuesta deja evidencias que el ejercicio profesional de la ingeniería se sustenta en una serie de habilidades que deberían representar fortalezas para los ingenieros.

Según (Tribuno, 2020) El escritor y novelista estadounidense James Albert Michener dijo: "Los científicos sueñan con hacer grandes cosas; los ingenieros simplemente las hacen".

Una de las evidencias de lo indicado fue el diseño y construcción de la torre de Eiffel obra de Alexandre Gustave Eiffel. Según (Tribuno, 2020) Thomas Edison le escribió: "Para el 
ingeniero Mr. Eiffel, el esforzado constructor de esta gigantesca y original muestra de ingeniería moderna, de alguien que tiene el mayor respeto y admiración por todos los ingenieros, incluyendo al Gran Ingeniero, el Buen Dios". Firmado Thomas Edison.

Según (Unitec, 2021) Ingenieros: no puedes vivir sin ellos, punto

Los ingenieros han dado forma al mundo que se conoce hoy, y entre sus aportes se tienen: Edificios inteligentes, Viajes espaciales, Automóviles veloces, Dispositivos electrónicos, y muchas otras cosas.

El trabajo de los ingenieros es mejorar cada uno de los procesos que realiza el ser humano en su vida diaria, así como crear nuevas opciones que antes ni siquiera pensábamos que eran posibles. (Unitec, 2021). De otro lado según (Diario, 2021) los ingenieros, según la UNESCO, trabajan en la "comprensión, diseño, desarrollo, invención, innovación y el uso de materiales, máquinas, estructuras, sistemas y procesos para fines concretos".

Para cumplir con esta misión Los ingenieros deberán estar en la capacidad de interactuar y dar respuesta a problemas económicos, medioambientales y de desarrollo científicotecnológico, enfrentados por la sociedad contemporánea (Diario, 2021).

\subsection{Definición de las Habilidades blandas y sus aplicaciones}

La habilidad, es la capacidad de realizar determinadas actividades o tareas y aplicar el conocimiento técnico para resolver problemas. (Garita-González, 2020). De la misma forma según (Rao, 2014), las habilidades blandas son conocidas como habilidades para la vida, habilidades interpersonales, habilidades de empleabilidad y de inteligencia emocional.

Estas habilidades son las que contribuyen al éxito en la vida y en el mundo laboral. (Neri, Junio 2020)

Las habilidades blandas tienen que ver más con las personas que con los conocimientos que poseen. Por estas razones y otras más, las habilidades blandas deben de considerarse como una inversión. (Herrera, 2020).

Según (Neri, Junio 2020) comenta que las habilidades blandas son competencias intangibles que son complejas de medir y están vinculadas con las actitudes del individuo. Sea como fuere, son competencias que tienen una relación positiva con los niveles salariales de las personas. 
Para (Patron, 2019) ...las competencias son habilidades. No obstante, las habilidades blandas, también conocidas como socio emocionales o no cognitivas, están referidas a características como apertura a nuevas experiencias, trabajo en equipo, perseverancia de largo plazo, y autocontrol de corto plazo.

Según las investigaciones realizadas se presentan las Tablas siguientes:

- Tabla 1: Definiciones de competencias blandas.

- Tabla 2: Habilidades importantes según (Mark Keil, 2013).

- Tabla 3: Descripción de habilidades blandas.

- Tabla 4: Competencias blandas importantes en el gerente de proyecto según IPMA (2006).

\section{Tabla 1.}

\section{Definiciones de competencias blandas}

\begin{tabular}{|c|c|}
\hline Autores & Definiciones \\
\hline Alles (2008) & $\begin{array}{l}\text { Explica que existen competencias blandas y competencias duras, distinguiendo como } \\
\text { competencias blandas las habilidades de la personalidad, y como competencias duras, los } \\
\text { conocimientos técnicos, prácticos, mecánicos, específicos para una tarea o actividad. Martin } \\
\text { (2000), indica que las habilidades duras son aquellas objetivas con criterios de ciencia e } \\
\text { ingeniería, mientras que las blandas son las subjetivas y sociales. }\end{array}$ \\
\hline Beach (2011) & $\begin{array}{l}\text { Afirma que el gerente de proyectos debe alcanzar el éxito de los proyectos, por ello es } \\
\text { necesario precisar las habilidades fundamentadas en la sinergia bajo los principios intangibles } \\
\text { de la confianza, el arte de adaptación y el cultivar las habilidades blandas ya que estos tres } \\
\text { principios ayudarán a crear un ambiente en el cual la gente tenga la voluntad de } \\
\text { desempeñarse a sus niveles más altos }\end{array}$ \\
\hline Villar y Quiroga (2013) & $\begin{array}{l}\text { Definen como competencia la capacidad de una persona para hacer un trabajo } \\
\text { correctamente, distinguiendo en la competencia la combinación integral de conocimientos } \\
\text { prácticos y teóricos, habilidades desarrolladas con la experiencia y actitudes reflejadas por el } \\
\text { comportamiento y los valores que se utilizan para mejorar el rendimiento }\end{array}$ \\
\hline Wallace (2014) & $\begin{array}{l}\text { Destaca que las competencias en materia de gestión de proyectos abarcan actitudes, } \\
\text { características básicas de la personalidad y liderazgo, y la capacidad de dirigir el equipo de } \\
\text { un proyecto }\end{array}$ \\
\hline Ortega (2016) & $\begin{array}{l}\text { Las habilidades socioemocionales, habilidades sociales y emocionales, habilidades de } \\
\text { carácter o rasgos de personalidad, que, aunque la literatura actual tiende a evitar } \\
\text { deliberadamente referirse a ellos como "rasgos" puesto que se supone que los rasgos } \\
\text { generalmente permanecen invariables, mientras que las habilidades pueden ser } \\
\text { desarrolladas }\end{array}$ \\
\hline
\end{tabular}


Lledó (2017)

Ortega (2017)

Negociación y resolución de conflictos
Asevera que el director de proyecto es como un director de orquesta, donde actúa como líder del equipo para alcanzar los objetivos del proyecto

Las habilidades blandas son un conjunto de destrezas que permiten desempeñarse mejor en las relaciones laborales y personales. Marca que los especialistas en educación coinciden en que las habilidades técnicas se pueden enseñar mucho más fácilmente que las habilidades blandas; y enuncia que muchos empresarios, directivos y expertos en materia laboral especialmente capacitadores plantean que, si tiene trabajadores con una gran comunicación, negociación y habilidades interpersonales, éstos deben ser retenidos en su organización Hace referencia a los pasos de negociación: determinar el objetivo de la negociación, identificar problemas, actitudes, estrategias, planificar la posición de negociación y decidir el plan de acción.

Fuente. (Patron, 2019).

\section{Tabla 2.}

Habilidades Importantes según (Mark Keil, 2013)

\begin{tabular}{|c|c|c|}
\hline Categoría de Habilidades & Descripción & Habilidades \\
\hline Gestión de equipos & $\begin{array}{l}\text { Deben proporcionar liderazgo, motivar y } \\
\text { capacitar a los miembros del equipo para } \\
\text { que ejecuten el proyecto con éxito. }\end{array}$ & $\begin{array}{l}\text { - } \text { Capacidad para motivar a los miembros } \\
\text { - } \text { Habilidad para empoderar a futuros } \\
\text { líderes. } \\
\text { - Celebrando logros } \\
\text { - Liderazgo } \\
\text { - Colaboración } \\
\text { - Habilidad para unir diversos equipos. } \\
\text { - Habilidades de equipo virtual }\end{array}$ \\
\hline Comunicación & $\begin{array}{l}\text { La comunicación implica las habilidades } \\
\text { necesarias para comunicarse eficazmente } \\
\text { con los involucrados en el proyecto, incluye } \\
\text { escuchar e identificar los problemas que } \\
\text { surgen durante el curso del proyecto }\end{array}$ & $\begin{array}{l}\text { - Comunicación verbal } \\
\text { - Comunicación escrita } \\
\text { - Escuchar } \\
\text { - Habilidad para construir argumentos } \\
\text { persuasivos. }\end{array}$ \\
\hline Talentos de la gente & $\begin{array}{l}\text { Se requieren habilidades personales para } \\
\text { mantener buenas relaciones con las } \\
\text { personas asociadas con el proyecto, } \\
\text { incluye ejercer influencia sobre las } \\
\text { personas. }\end{array}$ & $\begin{array}{l}\text { - Construir una relación } \\
\text { - Comprender la psicología de las } \\
\text { personas. } \\
\text { - Manejo de conflictos } \\
\text { - Habilidades de la honesta } \\
\text { - Negociación }\end{array}$ \\
\hline
\end{tabular}




$\begin{array}{ll}\text { Características personales } & \begin{array}{l}\text { Características personales Características } \\ \text { personales que pueden ser inherentes a la }\end{array} \\ \begin{array}{ll}\text { naturaleza, pero que aún pueden ser } \\ \text { alimentadas }\end{array} & \begin{array}{l}\text { - Habilidad para manejar el estrés. } \\ \text { Buscar consenso }\end{array} \\ & \text { - Persistencia } \\ & \text { - Cooperación } \\ & \text { - Atención a los detalles }\end{array}$

Fuente. Elaboración propia

La gestión de proyectos requiere de personas con determinadas habilidades según (Patron, 2019) A partir de la importancia que Swasti (2015), atribuye a las habilidades blandas en el gerente para la gestión de proyectos son presentadas en la Tabla 3 (Patron, 2019)

\section{Tabla 3.}

\section{Descripción de las habilidades blandas.}

\begin{tabular}{|c|c|}
\hline Habilidades blandas & Descripción \\
\hline Comunicación & $\begin{array}{l}\text { Tono y estilo en que los comunicadores hablan a su audiencia, analizan los problemas } \\
\text { y desafíos con colegas y clientes por igual }\end{array}$ \\
\hline Motivación & $\begin{array}{l}\text { Alentar el equipo de trabajo para que tome la iniciativa y trabaje positivamente, } \\
\text { mostrando su compromiso en el proyecto }\end{array}$ \\
\hline Liderazgo & $\begin{array}{l}\text { Pilar de habilidad blandas, es la capacidad de comunicarse de manera efectiva y la } \\
\text { aptitud para motivarse a sí mismo y motivar a los demás }\end{array}$ \\
\hline Conciencia de sí mismo & $\begin{array}{l}\text { Aceptar responsabilidades por cualquier error, demuestra la voluntad de aprender y } \\
\text { progresar. la autoconciencia }\end{array}$ \\
\hline Trabajo en equipo & $\begin{array}{l}\text { Hace referencia a la planificación, cooperación y coordinación de cada persona, la } \\
\text { capacidad de trabajar de manera eficiente con otros y el respeto saludable de las } \\
\text { diferentes opiniones, costumbres y preferencias individuales }\end{array}$ \\
\hline $\begin{array}{l}\text { Capacidad de trabajar bajo la } \\
\text { presión y la gestión del tiempo }\end{array}$ & $\begin{array}{l}\text { Habilidad de mostrar una actitud decisiva, pensar con claridad, trabajar bajo presión, } \\
\text { dentro de los plazos al priorizar las tareas }\end{array}$ \\
\hline Flexibilidad & $\begin{array}{l}\text { Capacidad para evolucionar, cambiar de acuerdo con la situación, capacidad de } \\
\text { adquirir nuevas habilidades y enfrentar nuevas tareas }\end{array}$ \\
\hline $\begin{array}{l}\text { Negociación y resolución de } \\
\text { conflictos }\end{array}$ & $\begin{array}{l}\text { Hace referencia a los pasos de negociación: determinar el objetivo de la negociación, } \\
\text { identificar problemas, actitudes, estrategias, planificar la posición de negociación y } \\
\text { decidir el plan de acción. }\end{array}$ \\
\hline
\end{tabular}

Fuente. Elaboración propia.

De acuerdo con (Patron, 2019) Seleccionan los 15 elementos de competencia conductual del IPMA (International Project Management Association) (2006) considerando las 
más importantes en la gestión de proyectos, agrupados y descritos en la tabla 4 como las competencias blandas importantes en el gerente de proyectos.

Tabla 4.

Competencias blandas importantes en el gerente de proyecto según IPMA (2006)

\begin{tabular}{|c|c|}
\hline $\begin{array}{l}\text { Competencias blandas } \\
\text { según IPMA }\end{array}$ & Descripción \\
\hline Liderazgo & $\begin{array}{l}\text { Son patrones de comportamiento, así como a las actitudes sobre comunicación, } \\
\text { resolución de conflictos, crítica, trabajo en equipo, toma de decisiones y delegación. }\end{array}$ \\
\hline Compromiso y motivación & $\begin{array}{l}\text { El compromiso y la motivación del gerente del proyecto y los miembros del equipo } \\
\text { reflejan la aceptación personal de todos los individuos asociados con el proyecto }\end{array}$ \\
\hline Autocontrol & $\begin{array}{l}\text { El autocontrol o la autogestión reflejan un enfoque sistemático y disciplinado para } \\
\text { enfrentar la rutina diaria y las situaciones estresantes }\end{array}$ \\
\hline Asertividad & $\begin{array}{l}\text { Características personales Características personales que pueden ser inherentes a la } \\
\text { naturaleza, pero que aún pueden ser alimentadas }\end{array}$ \\
\hline Relajación & $\begin{array}{l}\text { Implica la capacidad de expresar puntos de vista de manera persuasiva y autoritaria } \\
\text { teniendo en cuenta su impacto en la toma de decisiones }\end{array}$ \\
\hline Apertura & $\begin{array}{l}\text { Se refiere al cultivo de un clima abierto entre individuos para beneficiarse de sus } \\
\text { aportes, sugerencias, preocupaciones e inquietudes, evitando la discriminación cultural. }\end{array}$ \\
\hline Creatividad & $\begin{array}{l}\text { Describe la capacidad de pensar y actuar de manera original e imaginativa para lograr } \\
\text { el éxito del proyecto }\end{array}$ \\
\hline Orientación & $\begin{array}{l}\text { Describe la atención del equipo del proyecto sobre los objetivos clave para obtener el } \\
\text { resultado óptimo para todas las partes involucradas }\end{array}$ \\
\hline Eficiencia & $\begin{array}{l}\text { Se refiere a la asignación y explotación eficiente de todos los recursos disponibles para } \\
\text { el proyecto }\end{array}$ \\
\hline Consulta & $\begin{array}{l}\text { Se centra en la toma racional de decisiones y la presentación de argumentos sólidos } \\
\text { para encontrar soluciones }\end{array}$ \\
\hline Negociación & $\begin{array}{l}\text { Se establecen los medios por los cuales las partes involucradas pueden resolver los } \\
\text { desacuerdos hacia una solución mutuamente satisfactoria }\end{array}$ \\
\hline Conflicto & $\begin{array}{l}\text { Se describirse como un momento de gran dificultad, que exige un análisis de riesgos y } \\
\text { una planificación de escenarios para manejar estos obstáculos }\end{array}$ \\
\hline Confiabilidad & Capacidad de cumplir con las especificaciones de tiempo y calidad del proyecto \\
\hline Valores & $\begin{array}{l}\text { La apreciación de los valores se basa en el respeto mutuo y en la receptividad de las } \\
\text { opiniones, juicios de valor y estándares éticos de los demás }\end{array}$ \\
\hline Ética & $\begin{array}{l}\text { Conducta moralmente aceptada que representa las libertades personales y } \\
\text { profesionales, así como los límites }\end{array}$ \\
\hline
\end{tabular}

Fuente. Elaboración propia

\subsection{Necesidad de las habilidades blandas en la formación de los ingenieros}

Según (Neri, Junio 2020) Se concluye que las instituciones educativas requieren trabajar en la implementación de talleres que refuercen las habilidades blandas, así como mantener el 
acercamiento con el sector empresarial para que los logren el fortalecimiento de las competencias interpersonales.

Según las investigaciones de (Neri, Junio 2020) Los países que prioricen las políticas educativas y brinden oportunidades para el desarrollo de competencias que son consideradas importantes para los sectores de la sociedad pueden crear el escenario para mejorar la productividad y el desarrollo económico. Este es el reconocimiento a la necesidad de contar con el talento humano capaz de insertarse y crecer profesionalmente en el mercado de trabajo coadyuvará a que las organizaciones logren progreso y bienestar para los individuos que en ellas participen

La empleabilidad de los egresados se ha convertido en un indicador de calidad para las universidades.

De acuerdo con Retnanto, Parsaei y Parsaei (2019), las escuelas que imparten programas de ingeniería requieren fortalecer las habilidades sociales en los estudiantes. No obstante, otro de los problemas que enfrentan las universidades y sus profesores es que no cuentan con una forma clara de evaluar las competencias blandas. (Neri, Junio 2020)

Particularmente, en las escuelas de ingeniería los estudiantes pasan mayor tiempo perfeccionando sus habilidades técnicas y en ocasiones dejan de lado las competencias que les permiten resolver problemas de otra índole. (Neri, Junio 2020). Ante esta problemática es necesario identificar las formas que más aprecian y las que generan mayor motivación en los estudiantes a la hora de aprender (Kumar y Hsiao, 2007).

Las habilidades sociales son cruciales para los ingenieros, ya que el entorno laboral en el que se desarrollan cada vez es más interdisciplinario y demanda mayor trabajo colaborativo. (Neri, Junio 2020)

Al respecto, Florea y Stray (2018) analizaron anuncios de empleos en 33 países y comprobaron que las empresas desean contratar ingenieros capaces de comunicarse, posean habilidades analíticas y resuelvan problemas. También determinaron que existen nuevas categorías de habilidades blandas como la ética en el trabajo, enfoque en el cliente y saber trabajar bajo presión. (Neri, Junio 2020).

Esto concuerda con lo señalado por Fadel y Groff (2019) cuando mencionan que las escuelas tienen que impulsar una educación que tenga como objetivos forjar el carácter de los nuevos ingenieros para que adquieran y fortalezcan virtudes, valores y una capacidad de tomar 
decisiones para llevar una vida equilibrada, incluso se requiere enseñar lo relacionado con la salud mental, la curiosidad, la resiliencia, la ética y el liderazgo. (Neri, Junio 2020)

Siguiendo a Dell'Aquila et al. (2017) y Lennox y Roos (2017), las competencias blandas o inteligencias interpersonales son aquellas que permiten la creación y desarrollo de relaciones positivas con los demás, además de ser componentes clave en la resolución positiva de conflictos. (Neri, Junio 2020)

Según (Neri, Junio 2020) se encontró que, conforme los estudiantes avanzan por semestre, su percepción sobre la adquisición de las habilidades blandas es positiva. Otro hallazgo es que los jóvenes que percibieron que un egresado percibe los mejores ingresos al mes son los que obtuvieron más puntos en las competencias blandas, mientras que los que piensan que los egresados que perciben menores ingresos son los que obtuvieron menos puntos. (Neri, Junio 2020)

En otra investigación de (Neri, Junio 2020) Se reconoce que los estudiantes de la universidad en España, a diferencia de los estudiantes mexicanos, son los que perciben de forma más positiva las competencias blandas adquiridas.

Como parte de la misma investigación se comprobó que los estudiantes españoles son lo que mejor califican la competencia de la comunicación, el procesamiento de información, son capaces de afrontar retos con facilidad, tienen la habilidad de trabajar en equipo (habilidad claves para los estudiantes), poseen iniciativa y decisión para resolver problemas, retroalimentan de forma correcta los errores, se relacionan con facilidad, son persuasivos e influyentes en las decisiones que toman las demás personas, llevan a cabo autoanálisis y autocríticas para seguir mejorando, cuestionan ideas tanto propias como de las personas que los rodean, se mantienen informados en las tendencias de los mercados laborales, tienen la habilidad de reaccionar con calma ante situaciones de adversidad y aprovechan al máximo los medios electrónicos. Todas estas habilidades son claves para el desempeño de los nuevos ingenieros. (Neri, Junio 2020)

Complementariamente Shakir (2009) enfatiza que en los programas de estudios se deben integrar las habilidades de comunicación, pensamiento crítico, habilidades para resolver problemas, trabajo en equipo, aprendizaje permanente, gestión de la información, habilidad emprendedora, ética y moral profesional y capacidad para liderar. (Neri, Junio 2020) 
Por esto, se concluye que las instituciones de educación superior deben redoblar esfuerzos para ayudar a los jóvenes a incrementar sus competencias socioemocionales. De acuerdo con lo expuesto y analizado, se recomienda hacer los estudios necesarios de las competencias socioemocionales que requieren los mercados laborales de los ingenieros con esta información se tendría que actualizar los programas de estudio y, de esta forma, tener egresados preparados para ingresar a un entorno plagado de competencia. (Neri, Junio 2020)

En consecuencia, las universidades, en tanto sistemas complejos propios del desarrollo de los individuos y de las sociedades, deben estar inmersas en estos cambios. Sin embargo, los planes de estudio de la educación superior continúan siendo rígidos e inflexibles, pues evalúan conocimiento técnico sin una visión integral de las habilidades, las destrezas y las actitudes. En consecuencia, se mantiene la evaluación memorística basada en la resolución de exámenes. (Garita-González, 2020)

Según (Garita-González, 2020) como ejes clave de la estrategia de incorporación de las habilidades blandas en el plan de estudio de los centros de formación de los nuevos ingenieros se tendría que considerar preponderantemente:

- Identificación de políticas de gestión académica que garanticen la capacidad humana y organizacional para el logro de objetivos referentes a las habilidades blandas como técnicas y complementarias en forma integral.

- Diseño de un modelo cuantificable. Es necesario considerar un proceso de mediciones de avance que determinen el grado de cumplimiento de objetivos y de fortalecimiento de las habilidades, incluso el análisis retrospectivo al final de cada ciclo lectivo.

- Necesidad de mejora continua. Se efectúa un análisis retrospectivo y de evaluación del modelo, con el fin de establecer acciones de mejora continua.

- Interrelación con el mercado laboral. Es el establecimiento de mecanismos de realimentación y de vinculación permanente con el sector industrial para tener claridad sobre el nivel de satisfacción respecto del perfil de egreso de los nuevos profesionales. (Garita-González, 2020)

Como conclusión se tiene que las escuelas tienen que reforzar los programas de estancias académicas con la industria, ya que los alumnos pueden experimentar los ambientes laborales y perder el posible miedo a resolver problemas y convivir con personas de mayor edad y experiencia. (Neri, Junio 2020) 
Finalmente, se recomienda que los modelos educativos para la formación de los nuevos profesionales de ingeniería tomen en cuenta la propuesta detallada en la figura 1.

\section{Figura 1.}

Estrategia enseñanza y aprendizaje bajo la concepción holística.

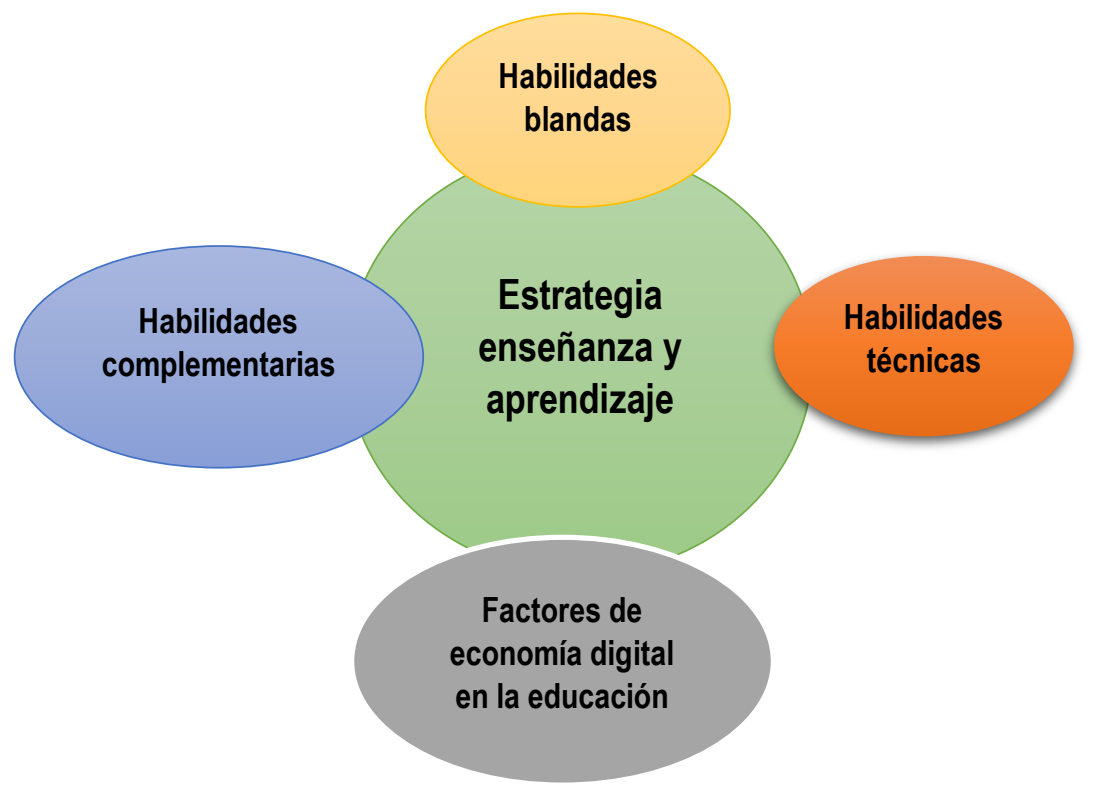

Fuente. Araya-Fernández y Garita-González (2019)

\subsection{Las habilidades blandas en la gerencia de proyectos}

En palabras de Garrido (2006), la gerencia es un proceso que coordina la planificación, organización, dirección y control de los recursos disponibles de una organización: humanos, físicos, tecnológicos y financieros; para lograr los objetivos previamente establecidos. (Patron, 2019) 
Los gerentes deben desarrollar habilidades para dirigir a los empleados, de manera tal que trabajen con eficiencia, pues la forma en que la gerencia ejerce su autoridad repercute en la productividad de la organización. (Patron, 2019)

En este sentido, Prieto (2014) afirma que la clave del éxito de la gestión de proyectos es que el director o gerente del proyecto desarrolle sus habilidades humanísticas en beneficio de los clientes o de la comunidad definida en el proyecto. (Patron, 2019)

En estudios realizados en el Perú se ha comprobado que existe una problemática clara sobre la alta frecuencia de fracasos en proyectos públicos y también privados. Esta situación genera la necesidad de analizar, estudiar la causalidad y tratar de averiguar si la falta de habilidades blandas o técnicas son las que serian que estarían generando esta problemática.

Por lo expuesto, nace una fuerte preocupación por determinar si la ausencia de habilidades blandas en la gerencia de proyectos encabezada por el equipo de supervisión es el factor que obstaculizaría el éxito de los proyectos de la institución en estudio. Los estudios de (Patron, 2019) explican que la ausencia de competencias blandas en la dirección de proyectos trae serias consecuencias. Denota un comportamiento en el que la gerencia culpa a los miembros del equipo y permite la presión de otros para cambiar objetivos, tareas o la especificación del proyecto tomando medidas correctivas de manera incorrecta

Por lo señalado por (Patron, 2019) los gerentes que no tienen las habilidades blandas gestionan el equipo como una máquina, cierra los ojos a los demás, parece a distancia, no realiza la gestión de las partes interesadas, evita los contactos con las partes involucradas y no tiene tiempo para la informalidad. La comunicación no se practica de forma fluida en ambos sentidos generando brechas entre miembros del equipo de supervisión y operadores, reflejándose en la baja motivación hacia la labor desarrollada. (Patron, 2019)

Los gerentes de proyectos de las organizaciones deben contar con habilidades blandas como el conjunto de rasgos o elementos de competencia personal como habilidades sociales, control del estrés, comunicación, motivación, entre otras cualidades para lograr el éxito en los proyectos. (Patron, 2019)

La negociación a cargo de gerentes sin competencias blandas puede bloquear la integración de los miembros de equipo para lograr acuerdos con los involucrados en el proyecto; en lo concerniente transferencia de recursos, generación de información y el cumplimiento de tareas (Patron, 2019) 
Las empresas cada vez invierten más energía y recursos en la formación de habilidades de sus directivos para que su autoridad no radique únicamente en su poder adquirido sino también en sus competencias para la gestión de proyectos (Patron, 2019), esta exigencia es estratégica. En este sentido, Prieto (2014) afirma que la clave del éxito de la gestión de proyectos es que el director o gerente del proyecto desarrolle sus habilidades humanísticas en beneficio de los clientes o de la comunidad definida en el proyecto. (Patron, 2019). Por este motivo, se genera la necesidad de investigar sobre la preocupación por determinar si la ausencia de habilidades blandas en la gerencia de proyectos encabezada por el equipo de supervisión es el factor que obstaculizar el éxito de los proyectos. (Patron, 2019)

La falta de habilidades blandas genera que la gerencia hable en tiempos inapropiados, critica, no motive a sus compañeros, aparece alejado del equipo ignorando a los demás, es pesimista, se angustia ante la presión, se alinea solo con la opinión mayoritaria. (Patron, 2019)

Por lo expuesto, se hace necesario que los gerentes de proyectos cuenten con habilidades blandas como el conjunto de los rasgos de competencia personal como habilidades sociales, control del estrés, comunicación, motivación, entre otras cualidades para promover el éxito en los proyectos. (Patron, 2019)

Como es sabido y sustentado según varios autores la ausencia de habilidades blandas en los gerentes de proyecto genera debilidad en la gestión, situación que conlleva a no lograr exitosamente las tareas a través de las personas que conforman el equipo (Patron, 2019)

La ausencia de las competencias blandas en la gerencia de proyectos conduce a desviaciones con lo planificado, personas desmotivadas, pérdida de recursos, conflictos injustificados, equipos disfuncionales, atraso en los resultados y pérdida de tiempo, porque estos factores determinan la productividad del proyecto (Patron, 2019)

El-Sabaa (2001), realizó una investigación sobre las habilidades humanas de gerentes de proyecto efectivo, en la cual evalúa cómo gerentes de proyecto y funcionales difieren con respecto a los atributos, habilidades y experiencias que se asocian con el desempeño exitoso de proyectos. (Patron, 2019)

Esto significa que, la habilidad humana se ocupa principalmente de trabajar con personas, es decir, un gerente de proyecto con una habilidad humana altamente desarrollada es suficientemente sensible a las necesidades y motivaciones de otros en su proyecto. (Patron, 2019) 
Finalmente, los resultados permitieron inferir las habilidades del gerente de proyectos, considerando que las habilidades de liderazgo son las más importantes pues, el gerente del proyecto debe ser un líder capaz de proporcionar una visión, demostrar carisma, y motivar a la gente hacia el logro de los objetivos del proyecto. (Patron, 2019)

Las habilidades de comunicación verbal fueron la segunda habilidad de más alto rango identificado, considerando que los gerentes de proyecto efectivos deben ser capaces de comunicarse con los diferentes grupos de interés para controlar el proyecto y resolver los problemas. (Patron, 2019).

Los resultados de esta investigación se consideran como antecedente al analizar y agrupar en orden de importancia las habilidades de liderazgo y comunicación, entre otras, en el gerente de proyecto. (Patron, 2019)

Se concluye que a nivel general los gerentes de proyectos del Instituto en estudio cuentan con las habilidades blandas (competencias personales y habilidades interpersonales) en un $45 \%$ y $52 \%$ respectivamente, que les permiten gestionar adecuadamente los proyectos asignados. (Patron, 2019)

\subsection{Las habilidades blandas en los niveles de gerencia}

En la actualidad, en los niveles de gerencia se evidencia algunas falencias en habilidades blandas lo cual genera problemas en decisiones y resultados.

Las organizaciones actualmente presentan debilidades en las habilidades blandas al interior de esta, como si fuera poco, la expansión de estas obliga a que los colaboradores estén en un relacionamiento constante con personas o empresas de diferentes orígenes. (Albañil, 2020)

Cuando un individuo llega a una posición que requiere de liderazgo, es donde se percatan de la necesidad de habilidades blandas, y una de las más importantes es la inteligencia emocional, ya que se tiene la formación académica, para el éxito se requiere implementar aquellas habilidades que fortalecen su proceso laboral y profesional (Albañil, 2020)

Las habilidades blandas requieren fortalecerse y se exploten de manera eficiente, ya que como lo concluye Sriruecha \& Buajan (2017) en su investigación las habilidades blandas contribuyen a que se logre los objetivos de la organización. (Albañil, 2020) 
Por lo que las personas en posiciones gerenciales deberían fortalecer las habilidades blandas ya que es vital en una organización, ya que, no es fácil conformar un equipo de trabajo y aún más trabajar en equipo, esto se convierte en un desafío (Albañil, 2020).

Es importante aprender a dinamizar para generar un ambiente laboral bueno, agradable y armónico, cuando no se logra es donde se originan conflictos, por la poca versatilidad que tienen algunas personas para aprender a escuchar y comunicarse con las mismas (Albañil, 2020)

Según Goleman (2010) la inteligencia emocional hace referencia a la gestión de la capacidad y habilidades psicológicas en las que se pueden ver involucradas las emociones en cuanto a sentimiento, control, modificación y manejo de las emociones propias, así como persuadir la de los demás. (Albañil, 2020). En la figura 2 se detalla la inteligencia emocional.

\section{Figura 2.}

\section{Elementos de la inteligencia emocional}

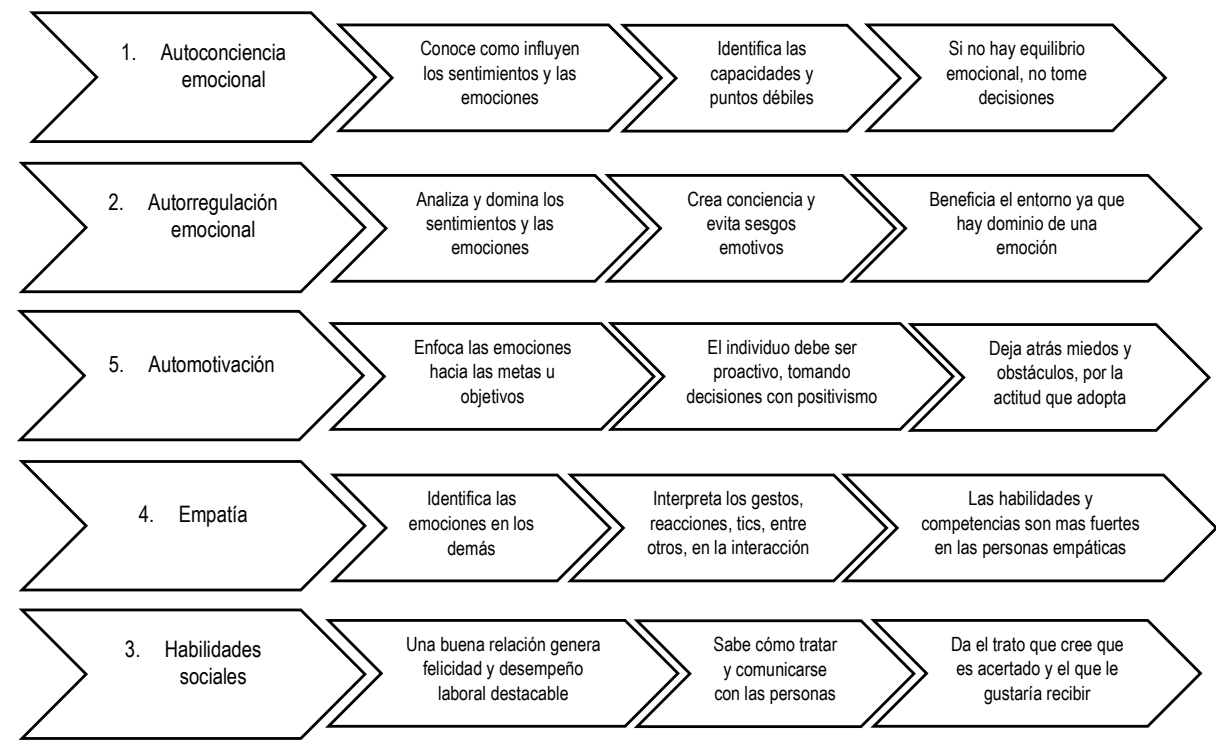

Fuente. (Albañil, 2020)

\section{HALLAZGOS}

De acuerdo con las investigaciones realizadas se aprecia que los ingenieros tienen un rol directo ante la sociedad. Por otro lado, los profesionales de ingeniería deben ejercer su profesión en diferentes facetas, dentro de las cuales se analizaron en primer lugar el ejercicio profesional directo como ingenieros, en segundo lugar, la gestión de proyectos, y en tercer lugar las funciones gerenciales. En todos estos roles se comprobó, no solo la necesidad de 
considerar las habilidades blandas en los programas de formación de los ingenieros, sino que de no considerarlo se afectaría su desempeño de los ingenieros en favor de la sociedad.

Otro de los hallazgos importantes, es que hay una correlación entre los resultados de la formación de los ingenieros que no cuentan con habilidades blandas en los resultados del desempeño de su ejercicio profesional. Como consecuencia de este hallazgo, se deduce la necesidad de que los Centros de Formación de ingeniería evalúen en que grado viene considerando las habilidades blandas como parte de sus proyecto y planes educativos.

Los temas, definiciones y consideraciones detalladas en las tablas 1, 2, 3 y 4 muestran información suficiente para su consideración en el autoanálisis por parte de los Centros de Formación de sus programas actuales y según los resultados puedan desarrollar rediseños de proyectos de formación profesional de ingeniería.

Quizá el hallazgo de la correlación entre la incorporación de habilidades blandas en la formación de la ingeniería y su impacto en la calidad de su desempeño podría ser considerado como el mas importante ya que podría significar la mejora que tendría significativo rol en la mejora del aporte de los ingenieros a la Sociedad.

\section{CONTRIBUCIONES}

Los resultados alcanzados por la investigación hacen evidente la necesidad de que las habilidades blandas sean incorporada en los programas de formación de los ingenieros en las instituciones a cargo de esta notable responsabilidad.

Como una contribución interesante es la propuesta de misión de los ingenieros en la que se detallan los tipos de competencias; por un lado, las competencias duras para desarrollar los proyectos que podrían generar la solución a los problemas de la sociedad, y por el otro lado, las competencias blandas requeridas para la fase de la gerencia de proyectos, y con esto tentar a proyectos exitosos. De esta forma se incrementaría la certidumbre de éxito de los proyectos y por lo tanto se mejoraría el aporte de la ingeniería a la sociedad.

Con los resultados de la presente investigación se tendría los sustentos necesarios para iniciar nuevas investigaciones:

a. ¿Qué hacer con los centros de formación de ingeniería, que no consideran las habilidades blandas e inteligencia emocional en sus proyectos educativos? 
b. ¿Qué tendrían que hacer los gremios empresariales relacionados a los proyectos en los cuales intervienen directamente los ingenieros?

c. ¿Qué tendría que hacer los gremios profesionales ante la necesidad de mejorar el desempeño de los ingenieros y la necesidad de las habilidades blandas?

d. ¿Qué preocupación tocaría a los propios ingeniería para mejorar sus habilidades como condiciones para mejorar su desempeño profesional?

\section{CONCLUSIONES}

Esta investigación cualitativa correlacional ha permitido validar la relación directa entre la necesidad de incorporación de las habilidades blandas, en sus diversas acepciones, detalladas en las Tablas 1, 2, 3 y 4 en la formación de los ingenieros como condición para mejorar su desempeño profesional y rol ante la sociedad.

Los ingenieros tienen tres facetas principales de aporte a la sociedad: trabajo como ingeniero, ejercicio profesional como gerente de proyectos, y desarrollo de puestos gerenciales para todas estas posiciones se ha validado la prioridad que representan "Las habilidades blandas, como factor clave para el desempeño de los ingenieros y mejora de aporte hacia la sociedad". Queda en responsabilidad de las personas que tengan incumbencia en la formación y dirección de las universidades la reflexión e implementación de las propuestas del presente trabajo.

\section{POSIBLES LIMITACIONES DEL PROYECTO}

Las limitaciones del proyecto están referidas especialmente a la conveniente de efectuar otras investigaciones relacionadas para dejar muy claramente el impacto de las propuestas en el potencial de desarrollo que se podrían generar los programas de formación de los ingenieros que consideren las propuestas señaladas en el presente trabajo.

\section{REFERENCIAS}

Albañil, E. A. (2020). Estrategias de fortalecimiento en habilidades blandas en el nivel directivo. Bogota, Colombia: Eespecializacion en alta gerencia Universidad Militar Nueva Granada Facultad de Ciencias Economicas.

Diario, E. (23 de Abril de 2021). El peridoico de Pereira. Obtenido de https://www.eldiario.com.co/contenido-patrocinado/el-mundo-necesita-masingenieros-las-ingenierias-el-motor-que-impulsa-la-mayoria-de-los-cambiostecnologicos-en-la-sociedad/ 
Garita-González, E. A.-F. (2020). Habilidades blandas: elementos para una visión holistica en la formacion de profesionales de informatica. Trilogía Ciencia Tecnología Sociedad, 11-36.

Herrera, C. A. (2020). Las habilidades blandas en estudiantes de ingenieria de tres instituciones publicas de educacion superior. RIDE.

María Elena Zepeda-Hurtado, E. O.-E.-B. (23 octubre 2018 de Octubre de 2018). redalyc.org. Obtenido de https://www.redalyc.org/jatsRepo/614/61458265007/html/index.html

Mark Keil, H. K. (2013). Understanding the most critical skills for managing IT projects: A Delphi study of IT project managers. Information \& Mangement, 398414.

Neri, C. A. (Junio 2020). Las habilidades blandas en estudiantes de ingeniería de tres instituciones públicas de educación superior. RIDE.

Patron, J. I. (2019). Competencias blandas como fundamento para el exito en la gestion de proyectos en la gerencia del Instituto de Proteccion Social del Sector Publico de Cartagena. Cartagena de Indias,.

Rao, M. S. (2014). Enhancing employability in engineering and management students.

Tribuno, E. (30 de Junio de 2020). La ingeniería es clave para las soluciones a todos los desafios. Obtenido de https://www.eltribuno.com/salta/nota/2020-6-30-0-0-0-laingenieria-es-clave-para-las-soluciones-a-todos-los-desafios

Unitec. (12 de Enero de 2021). Unitec/Blog. Obtenido de https://blogs.unitec.mx/vidauniversitaria/por-que-el-mundo-necesita-mas-ingenieros/

Vanegas, C. A. (2019). Habilidades blandas necesarias para la formación del ingeniero de sistemas del siglo XXI. Bogota: Universidad Piloto de Colombia Unidad Académica de Ciencias de la Educación Especialización en Docencia Universitaria. 